\title{
Fungi on irrigated rice seeds produced in the pre-germinated system in the Alto Vale do Itajaí region, Santa Catarina state, Brazil
}

\author{
Bruno Tabarelli Scheidt ${ }^{1^{*}}$ (iD) Juliano Berghetti ${ }^{1}$ (iD) Flávio Chupel Martins ${ }^{1}$ (iD \\ Ricardo Trezzi Casa ${ }^{1}$ (D) Welliton Recalcatti ${ }^{2}$ (D) Valdemir Rossarola ${ }^{2}$ (ID) \\ José de Alencar Lemos Vieira Junior ${ }^{3}$ (D)
}

${ }^{1}$ Programa de Pós-graduação em Produção Vegetal (PPGPV), Universidade do Estado de Santa Catarina (UDESC), 88520-000, Lages, SC, Brasil. E-mail: brunotabarelli.s@hotmail.com. *Corresponding author.

${ }^{2}$ Universidade do Estado de Santa Catarina (UDESC), Lages, SC, Brasil.

${ }^{3}$ Universidade do Oeste de Santa Catarina (UNOESC), Campos Novos, SC, Brasil.

ABSTRACT: The aim of this research was to identify and quantify fungi infecting irrigated rice seeds produced in the 2015/16, 2016/17 and 2017/18 harvests in the pre-germinated system in the Alto Vale do Itajai Region, Santa Catarina State, Brazil. A total of 479 lots of eight cultivars were analyzed. Seeds were disinfected and sown in potato-sucrose-agar culture medium with incubation for seven days at $25^{\circ} \mathrm{C} \pm$ $2^{\circ} \mathrm{C}$ and photoperiod of 12 hours. The main fungus detected in the three crops season was Microdochium albescens with 54.9\% of average incidence, followed by Alternaria padwikii (7.7\%) and Bipolaris oryzae (3.3\%).

Key words: Microdochium albescens, Oryza sativa, seed health.

Fungos em sementes de arroz irrigado produzidas no sistema pré-germinado na região do Alto Vale do Itajaí, estado de Santa Catarina, Brasil

RESUMO: O objetivo deste trabalho foi identificar e quantificar fungos infectando sementes de arroz irrigado, produzidas nas safras 2015/16, 2016/17 e 2017/18, no sistema pré-germinado na região do Alto Vale do Itajaí, estado de Santa Catarina. Foram analisados 479 lotes de oito cultivares, sendo as sementes desinfestadas e semeadas em meio de cultura de batata-sacarose-ágar com incubação por sete dias a $25^{\circ} \mathrm{C} \pm 2$ ${ }^{\circ} \mathrm{C}$ e fotoperiodo de 12 horas. O principal fungo detectado nas três safras foi Microdochium albescens com 54,9\% de incidência média, seguido de Alternaria padwikii (7,7\%) e Bipolaris oryzae (3,3\%).

Palavras-chave: Microdochium albescens, Oryza sativa, sanidade de sementes.

In the state of Santa Catarina (SC), irrigated rice (Oryza sativa L.) is produced in a pregerminated system widely sown in monoculture areas and in regions where long periods of wetness occur, factors that favor the occurrence of diseases (SOSBAI, 2018). In this crop system the pathogenic fungus inoculum is easily dispersed to the panicles with subsequent infection of grains or seeds, which can cause stains and their reduction (MIURA, 2002).

Fungi Alternaria padwickii (Ganguly), Bipolaris oryzae (Haan's Breda), Cercospora janseana (Racib.), Fusarium moniliforme (Sheldon), Microdochium albescens (Thüm.) syn. Microdochium oryzae; Gerlachia oryzae (Hashioka \& Yokogi), Pyricularia oryzae (Cav.) and Sarocladium oryzae (Saw) some of the main pathogens associated with rice seed
(MEW \& GONZALES, 2002; SOSBAI, 2018). Infected seeds may affect physiological quality and constitute an important agent of pathogen dissemination (OU, 1972).

Rice seed production in SC focuses mainly on the pursuit of high physiological quality. There is no obvious concern for seed production with high health quality or low incidence of pathogens. The aim of this research was to identify and quantify fungi infecting seeds of irrigated rice cultivars produced in the pre-germinated system in the Alto Vale do Itajaí (AVI), SC, Brazil.

Seed samples produced in the $2015 / 16$, 2016/17 and 2017/18 crops season, benefited by Cooperative Cravil, were analyzed in a total of 479 lots of SCS121 CL (165 lots), SCS122 Miura (85), SCS116 Satoru cultivars. (79), EPAGRI 109 (58), 
SCSBRS Tio Taka (49), SCS118 Marquês (22), SCS117 CL (11) and Primoriso CL (10), from the AVI region, SC. Disease management in these crops followed the indications of the cooperative. For chemical control, a mixture of strobirulin + triazole + benzothiazole was sprayed at the stages of booting and flowering and / or grain formation (SOSBAI, 2018). In the cooperative the seeds were stored in polypropylene bags with humidity and room temperature. Seeds were collected and sent to the Plant Pathology Laboratory Santa Catarina State University (UDESC). Detection of fungi was done in PSA + A culture medium $($ Potato-Sucrose-Agar + Antibiotic $=$ $200 \mathrm{mg} \mathrm{L}^{-1}$ streptomycin sulfate). Seeds were disinfected with sodium hypochlorite solution (1\%) for two minutes and then washed with sterile water. For each lot, 200 seeds were analyzed, four replicates of 50 , sown in acrylic Petri dishes and kept in growth chambers for seven days at $25^{\circ} \mathrm{C}$ and 12 hours photoperiod. For detection of $P$. oryzae the paper substrate incubation method (blotter test) was used. Six lots were randomly selected from each cultivar, totaling 48 lots. The seeds were arranged in a gerbox acrylic box containing two layers of moistened filter paper and incubated in a continuous light growth chamber at $27{ }^{\circ} \mathrm{C}$. The evaluation was performed at four and seven days. A completely randomized experimental design was used for both detection methods. Were considered infected in which it was possible to identify the colony and / or structures of the fungi, confirming the identification with microscope (BARNETT \& HUNTER, 1998).

The main pathogenic fungus associated with seeds were $M$. albescens with $54.9 \%$ of the average incidence and $100 \%$ of the prevalence, respectively followed by $A$. padwikii $(7.7 \% / 90.6 \%)$, B. oryzae $(3.3 \%$ / $59.9 \%)$, Curvularia sp. $(3.1 \%$ / $66.5 \%)$ and $S$. oryzae $(2.7 \% / 49.4 \%)$ (Table 1 and

Table 1 - Average incidence of fungi associated with seeds of irrigated rice cultivars produced in the Alto Vale do Itajaí Region, Santa Catarina State. Lages, 2019

\begin{tabular}{|c|c|c|c|c|c|c|c|c|c|c|c|c|}
\hline \multirow[t]{2}{*}{ Cultivar } & \multirow[t]{2}{*}{$\mathrm{N}^{\mathrm{o}}$ Lotes } & \multicolumn{11}{|c|}{-----------------------------------------------Incidence of fungi (\%)-------------------------------------------- } \\
\hline & & Mic & Alt & Cur & Bip & Sar & Nig & Pen & Asp & Altsp & $\mathrm{Cer}$ & Fus \\
\hline \multicolumn{13}{|c|}{-----------------------------------------------------------------------Safra 2015 / 2016 } \\
\hline SCS121 CL & 57 & 61.9 & 6.0 & 6.6 & 7.3 & 2.2 & 0.8 & 1.1 & 0.7 & 0.1 & 0.2 & 0.0 \\
\hline SCS116 Satoru & 33 & 72.3 & 2.9 & 1.7 & 1.6 & 2.2 & 1.6 & 1.3 & 1.2 & 0.4 & 0.3 & 0.0 \\
\hline SCSBRS Tio Taka & 17 & 55.6 & 7.7 & 5.7 & 7.3 & 2.7 & 3.0 & 0.5 & 0.7 & 0.9 & 0.4 & 0.4 \\
\hline Epagri 109 & 11 & 68.1 & 2.0 & 2.0 & 2.5 & 3.9 & 1.2 & 2.1 & 2.3 & 1.0 & 0.0 & 0.0 \\
\hline SCS117 CL & 11 & 60.5 & 9.0 & 3.9 & 5.1 & 2.5 & 1.4 & 0.4 & 0.3 & 0.5 & 0.4 & 0.0 \\
\hline SCS118 Marquês & 10 & 57.2 & 7.6 & 9.6 & 3.3 & 3.7 & 0.7 & 1.8 & 1.8 & 0.0 & 0.2 & 0.0 \\
\hline Average (\%) & & 62.6 & 5.8 & 4.9 & 4.5 & 2.8 & 1.4 & 1.2 & 1.2 & 0.5 & 0.3 & 0.1 \\
\hline \multicolumn{13}{|c|}{ - } \\
\hline SCS121 CL & 55 & 530 & 7.4 & 3.0 & 2.7 & 4.2 & 0.9 & 0.6 & 1.0 & 0.7 & 1.2 & 0.1 \\
\hline SCS116 Satoru & 33 & 506 & 9.1 & 2.4 & 2.8 & 2.7 & 0.7 & 1.3 & 1.9 & 0.9 & 0.8 & 0.2 \\
\hline Epagri 109 & 31 & 455 & 11.3 & 3.0 & 3.2 & 3.9 & 0.6 & 0.8 & 1.3 & 1.5 & 0.6 & 0.3 \\
\hline SCSBRS Tio Taka & 26 & 627 & 6.5 & 2.5 & 3.4 & 3.4 & 0.7 & 0.5 & 0.7 & 1.0 & 0.5 & 0.3 \\
\hline SCS122 Miura & 12 & 431 & 11.7 & 5.6 & 5.1 & 7.1 & 0.0 & 1.2 & 2.1 & 2.3 & 1.1 & 0.0 \\
\hline SCS118 Marquês & 12 & 453 & 9.3 & 3.0 & 3.3 & 4.8 & 1.6 & 0.4 & 0.8 & 1.6 & 1.1 & 0.0 \\
\hline Average $(\%)$ & & 500 & 9.2 & 3.2 & 3.4 & 4.3 & 0.7 & 0.8 & 1.3 & 1.3 & 0.9 & 0.2 \\
\hline \multicolumn{13}{|c|}{ 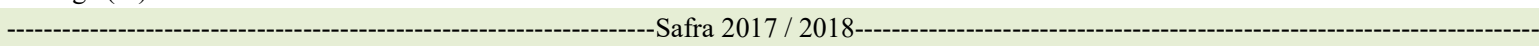 } \\
\hline SCS122 Miura & 73 & 535 & 7.2 & 1.7 & 1.6 & 1.1 & 0.6 & 0.1 & 0.0 & 0.3 & 1.2 & 0.0 \\
\hline SCS121 CL & 53 & 516 & 5.9 & 0.8 & 3.3 & 0.5 & 0.8 & 0.1 & 0.2 & 0.4 & 1.0 & 0.1 \\
\hline Epagri 109 & 16 & 446 & 14.3 & 1.7 & 1.9 & 0.3 & 0.7 & 0.1 & 0.0 & 0.8 & 1.2 & 0.0 \\
\hline SCS116 Satoru & 13 & 489 & 8.9 & 1.2 & 2.6 & 0.3 & 0.0 & 0.2 & 0.4 & 0.7 & 2.5 & 0.1 \\
\hline Primoriso CL & 10 & 577 & 7.2 & 1.0 & 0.9 & 0.9 & 0.5 & 0.2 & 0.0 & 0.4 & 2.8 & 0.0 \\
\hline SCSBRS Tio Taka & 6 & 562 & 4.5 & 0.7 & 2.5 & 2.2 & 0.3 & 0.3 & 0.0 & 0.3 & 1.2 & 0.0 \\
\hline Average (\%) & & 521 & 8.0 & 1.2 & 2.1 & 0.9 & 0.5 & 0.2 & 0.1 & 0.5 & 1.6 & 0.0 \\
\hline Overall Average (\%) & & 550 & 7.7 & 3.1 & 3.3 & 2.7 & 0.9 & 0.7 & 0.9 & 0.8 & 0.9 & 0.1 \\
\hline
\end{tabular}

Mic - Microdochium albescens; Alt - Alternaria padwiicki; Cur - Curvularia sp.; Bip - Bipolaris oryzae; Sar - Sarocladium oryzae; Nig Nigrospora oryza; Pen - Penicillium spp; Asp - Aspergillus flavus; Alt ${ }_{\mathrm{sp}}$ - Alternaria sp.; Cer - Cercospora janseana e Fus - Fusarium sp. 
table 2). Similar results were reported in irrigated rice cultivars in 350 samples analyzed in Rio Grande do Sul State (RS), from 1993 to 1998 , also detecting G. oryzae (18.0\%), Alternaria sp. (6.3\%), C. lunata (4.9\%) and $B$. oryzae $(2.6 \%)$ (FRANCO et al., 2001). In another study also in RS, in 162 plots of rice from the 2005/06 crop season, Alternaria sp. (9.6\%), Bipolaris sp. (9.3\%), Gerlachia sp. (4.9\%) and Curvularia sp. (3.8\%) (FARIAS et al., 2007).

The fungus $M$. albescens was the only with $100 \%$ prevalence (Table 2). This pathogen causes scalding in rice leaves, a growing disease in crop in the AVI, SC. The fungus was reported in RS association with seeds ranging from 6 to $31 \%$ (average 18\%) (FRANCO et al., 2001) and 1 to $33 \%$ (average 4.9\%) (FARIAS et al., 2007), but with a lower incidence than that obtained in this study (range 43.1 to $72.3 \%$ and average $54.9 \%$ ). This may be related to the detection method, reaction of rice genotypes and cropping systems. MALAVOLTA (2007), using filter paper method detected an average incidence of M. oryzae of 24.6 and $29.8 \%$, respectively in 2003 and 2004, in rice genotype seeds in the of São Paulo (SP) state. PSA medium is more efficient in detecting $M$. albescens when compared to the filter paper method (GUTIÉRREZ et al. 2009). Analyzing genotypes, a higher incidence was observed in SCS116 Satoru (72.3\% in 2015/16 crop) and lower in SCS122 Miura ( $43.1 \%$ in $2016 / 17$ crop). These values are considered high for a possible obtaining of healthy seed for pathogen management, since there is no information on resistance reaction of cultivars for $M$. albescens in the states of SC and RS (SOSBAI, 2018).

The fungus $A$. padwickii, included in the group of grain spotters, obtained average incidence in the three harvests of $7.7 \%$ and prevalence of

Table 2 - Average prevalence of fungi associated with seeds of irrigated rice cultivars produced in the Alto Vale do Itajaí Region, Santa Catarina State. Lages, 2019.

\begin{tabular}{|c|c|c|c|c|c|c|c|c|c|c|c|c|}
\hline \multirow[t]{2}{*}{ Cultivar } & \multirow[t]{2}{*}{$\mathrm{N}^{\mathrm{o}}$ Lotes } & \multicolumn{11}{|c|}{---Prevalence of fungi (\%)-- } \\
\hline & & Mic & Alt & Cur & Bip & Sar & Nig & Pen & Asp & Altsp & Cer & Fus \\
\hline \multicolumn{13}{|c|}{ 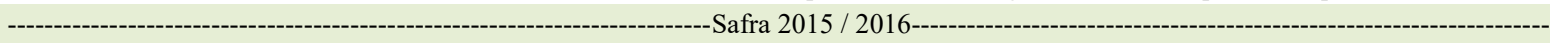 } \\
\hline SCS121 CL & 57 & 100 & 84.7 & 66.7 & 39.4 & 63.2 & 36.4 & 33.3 & 48.5 & 21.2 & 9.1 & 0.0 \\
\hline SCS116 Satoru & 33 & 100 & 100 & 70.6 & 76.5 & 57.6 & 64.7 & 29.4 & 23.5 & 17.7 & 17.7 & 0.0 \\
\hline SCSBRS Tio Taka & 17 & 100 & 100 & 90.9 & 45.5 & 64.7 & 54.6 & 36.4 & 9.1 & 18.2 & 18.2 & 0.4 \\
\hline Epagri 109 & 11 & 100 & 36.4 & 45.5 & 54.6 & 81.8 & 27.3 & 54.6 & 63.6 & 27.3 & 9.1 & 0.0 \\
\hline SCS117 CL & 11 & 100 & 89.5 & 89.5 & 77.2 & 72.7 & 26.3 & 45.6 & 22.8 & 8.8 & 14.0 & 0.0 \\
\hline SCS118 Marquês & 10 & 100 & 90.0 & 100 & 50.0 & 70.0 & 40.0 & 50.0 & 40.0 & 0.0 & 100 & 0.0 \\
\hline Average (\%) & & 100 & 83.4 & 77.2 & 57.2 & 68.3 & 41.6 & 41.6 & 34.6 & 15.5 & 13.0 & 0.1 \\
\hline \multicolumn{13}{|c|}{ 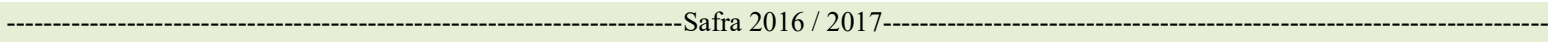 } \\
\hline SCS121 CL & 55 & 100 & 89.1 & 80.0 & 63.6 & 63.6 & 23.6 & 27.3 & 36.4 & 32.7 & 40.0 & 3.6 \\
\hline SCS116 Satoru & 33 & 100 & 90.9 & 69.7 & 60.6 & 45.5 & 21.2 & 48.5 & 42.4 & 36.4 & 21.2 & 6.1 \\
\hline Epagri 109 & 31 & 100 & 92.3 & 69.2 & 53.8 & 38.5 & 19.2 & 21.9 & 19.2 & 46.2 & 34.6 & 7.7 \\
\hline SCSBRS Tio Taka & 26 & 100 & 96.8 & 77.4 & 67.7 & 58.1 & 16.1 & 32.3 & 45.2 & 54.8 & 32.3 & 9.7 \\
\hline SCS122 Miura & 12 & 100 & 100 & 91.7 & 100 & 91.7 & 0.0 & 58.3 & 50.0 & 75.0 & 33.3 & 16.7 \\
\hline SCS118 Marquês & 12 & 100 & 100 & 91.7 & 75.0 & 50.0 & 50.0 & 16.2 & 25.0 & 50.0 & 25.0 & 16.7 \\
\hline Average (\%) & & 100 & 94.8 & 79.9 & 70.1 & 57.9 & 21.7 & 34.1 & 36.4 & 49.2 & 31.1 & 10.1 \\
\hline \multicolumn{13}{|c|}{ 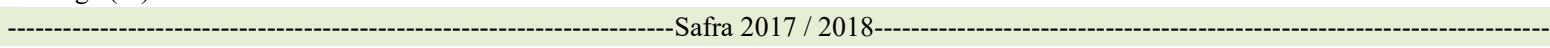 } \\
\hline SCS122 Miura & 73 & 100 & 868 & 26.4 & 66.0 & 18.9 & 22.6 & 5.7 & 7.6 & 18.9 & 32.1 & 5.7 \\
\hline SCS121 CL & 53 & 100 & 100 & 53.1 & 69.2 & 15.4 & 23.1 & 15.4 & 15.4 & 30.8 & 53.8 & 0.0 \\
\hline Epagri 109 & 16 & 100 & 100 & 16.6 & 50.0 & 0.0 & 16.7 & 16.7 & 0.0 & 16.7 & 50.0 & 0.0 \\
\hline SCS116 Satoru & 13 & 100 & 937 & 50.0 & 56.3 & 12.5 & 18.8 & 6.3 & 0.0 & 31.3 & 50.0 & 0.0 \\
\hline Primoriso CL & 10 & 100 & 918 & 57.5 & 45.2 & 34.3 & 12.3 & 6.9 & 1.4 & 10.9 & 49.3 & 2.7 \\
\hline SCSBRS Tio Taka & 6 & 100 & 90.0 & 50.0 & 30.0 & 50.0 & 0.0 & 10.0 & 0.0 & 20.0 & 90.0 & 0.0 \\
\hline Average (\%) & & 100 & 93.7 & 42.3 & 52.8 & 21.9 & 15.6 & 10.2 & 4.1 & 21.4 & 54.2 & 1.4 \\
\hline Overall Average (\%) & & 100 & 90.6 & 66.5 & 59.9 & 49.4 & 26.3 & 28.6 & 25.0 & 28.7 & 32.8 & 11.6 \\
\hline
\end{tabular}

Mic - Microdochium albescens; Alt - Alternaria padwiicki; Cur - Curvularia sp.; Bip - Bipolaris oryzae; Sar - Sarocladium oryzae; Nig -

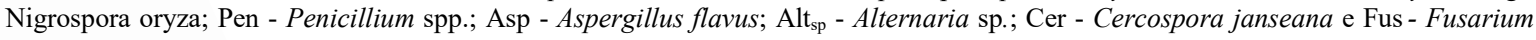
sp. 
90.6\%. In Egypt, India, Korea, Nepal and Thailand, the fungus was detected in 282 lots of 388 analyzed (MATHUR et al., 1972). In Brazil, in SP in 2003 and 2004, the average incidence of $A$. padwickii was $8.2 \%$ and $1.6 \%$; respectively, in cultivation of genotypes in flood irrigated system (MALAVOLTA, 2007). In RS, FRANCO et al. (2001), detected values from $0.4 \%$ to $20.6 \%$, but do not describe the Alternaria species.

The fungus $B$. oryzae presented higher average incidence in cultivar SCS121 CL and SCSBRS Tio Taka (7.3\%, 2015/16 crop), and lower average value in cultivar Primoriso CL $(0.9 \%$, $2017 / 18$ crop). For this fungus, we highlighted a prevalence of $100 \%$ detected in the $2016 / 17$ crop in the cultivar SCS122 Miura. In SP was detected an average incidence of $13.6 \%$, higher than that found in this study (MALAVOLTA, 2007).

The fungus Curvularia sp. was detected with incidence and prevalence values similar to $B$. oryzae. It was not possible to identify the species of Curvularia since no morphological and molecular characterization was performed. The species $C$. eragrostidis (P. Henn) and C. lunata (Wakker) Boedijn, are detected in rice seeds (LIMA \& FURTADO, 2007). Other fungi were detected with lower average incidence, such as: S. oryzae (2.7\%), Nigrospora oryzae $(0.9 \%)$, Penicillium spp. (0.7\%), Aspergillus flavus (0.9\%), Alternaria sp. (0.8\%), C. janseana (0.9\%) and Fusarium sp. (0.1\%) (Table 1). These are also reported in seeds in RS (FRANCO et al., 2001; FARIAS et al., 2007). The fungus $P$. oryzae was not detected by the tested. In RS P. oryzae was detected with low incidence $(0.04 \%)$ in 350 seed samples (FRANCO et al., 2001), but later was not detected in 162 samples analyzed (FARIAS et al., 2007).

This was the first survey of fungi associated with irrigated rice seeds in the pre-germinated cultivation system carried out in Santa Catarina State, serving as a basis for future research related to the epidemiological importance of infected seeds.

\section{ACKNOWLEDGEMENTS}

The reserach was financed in part by the Coordenação de Aperfeiçoamento de Pessoal de Nível Superior - Brasil (CAPES) - Finance code 001.

\section{DECLARATION OF CONFLICT OF INTERESTS}

The authors declare no conflict of interest. The founding sponsors had no role in the design of the study; in the collection, analyses, or interpretation of data; in the writing of the manuscript, and in the decision to publish the results.

\section{AUTHORS' CONTRIBUTIONS}

BTS and RTC conceived and designed experiments. BTS, VR and FCM performed the experiments, BTS carried out the lab analyses. BTS, JALVJ e JB performed statistical analyses of experimental data. BTS, JB, RTC, FCM, WR, VR and JALVJ prepared the draft of the manuscript. All authors critically revised the manuscript and approved of the final version.

\section{REFERENCES}

BARNETT, H. L.; HUNTER, B. B. Illustred genera of imperfect fungi. The American Phytopathological Society. St. Paul. 1998. 218p.

FARIAS, J. R. C. et al. Incidência de fungos associados a sementes de arroz em seis regiões produtoras do Rio Grande do Sul. Revista Brasileira de Agrociência, v.14, p.487-490, 2007. Available from: $<$ https://periodicos.ufpel.edu.br/ojs2/index.php/CAST/article/ viewFile/1395/1381>. Accessed: Jan, 30, 2020.

FRANCO, D. F. et al. Fungos associados a sementes de arroz irrigado no Rio Grande do Sul. Revista Brasileira de Agrociência, v.7, p.235-236, 2001. Available from: <http://www.ufpel.tche.br/ faem/agrociencia/v7n3/artigo16.pdf $>$. Accessed: Jan. 30, 2020.

GUTIÉRREZ S. A. et al. Estudio preliminar sobre métodos de detección de Microdochium oryzae em semillas de arroz. Tropical Plant Pathology, v.34, p.042-044, 2009. Available from <http://www.scielo.br/scielo.php?script=sci arttext\&pid=S198256762009000100007\&lng=en\&nrm=iso $>$. Accessed:Feb. 06, 2020. doi: 10.1590/S1982-56762009000100007.

LIMA, A. B.; FURTADO, M. Curvularia species (anamorphic fungi: Hyphomycetes) from Santiago island, Cape Vert. Portugaliae Acta Biologica, v.22, p.145-156, 2007. Available from: <https:// www.cabdirect.org/cabdirect/abstract/20083133955>. Accessed: Feb. 23, 2020

MALAVOLTA, V. M. A. et al. Incidência de fungos e quantificação de danos em sementes de genótipos de arroz. Summa Phytopathologica, v.33, p.280-286, 2007. Available from: $<$ https://www.scielo.br/scielo.php?pid=S0100540520070003000 12\&script $=$ sci_abstract\&tlng=pt $>$. Accessed: Feb. 25, 2020. doi: 10.1590/S0100-54052007000300012.

MATHUR, S. B. et al. Seed-borne infection of Trichoconis padwickii in rice, distribution, and damage to seeds and seedlings. International Seed Testing Association, v.37, p.803-810, 1972.

MEW, T. W.; GONZALES, P. A handbook of rice seedborne fungi. Los Baños, (Philippines): International Rice Research Institute. Science Publishers, Inc. 2002, 83p.

MIURA, L. Doenças. In: Epagri Arroz irrigado: Sistema prégerminado. Florianóplis, Epagri/ GMC, 2002, 203-227p.

OU, S. H. Rice diseases. Surrey: Commonwealth Mycological Institute, 1972. 368p.

SOSBAI. Arroz irrigado: recomendações técnicas da pesquisa para o Sul do Brasil. In: XXXII Reunião Técnica da Cultura do Arroz Irrigado, 2018, Farroupilha, RS. Cachoeirinha: Sociedade Sul-Brasileira de Arroz Irrigado, 2018, 205p. Available from: $<$ http://www.sosbai.com.br/docs/Boletim_RT 2016.pdf $>$. Accessed: Jan. 22, 2020. 


\section{Erratum}

In the article "Fungi on irrigated rice seeds produced in the pre-germinated system in the Alto Vale do Itajaí region, Santa Catarina state, Brazil" published in Ciência Rural, volume 50, number 8, DOI http://doi.org/10.1590/0103-8478cr20190903.

\section{In the table 1, where we read:}

Table 1 - Average incidence of fungi associated with seeds of irrigated rice cultivars produced in the Alto Vale do Itajaí Region, Santa Catarina State. Lages, 2019.

\begin{tabular}{|c|c|c|c|c|c|c|c|c|c|c|c|c|}
\hline \multirow[t]{2}{*}{ Cultivar } & \multirow[t]{2}{*}{$\mathrm{N}^{\mathrm{o}}$ Lotes } & \multicolumn{11}{|c|}{ 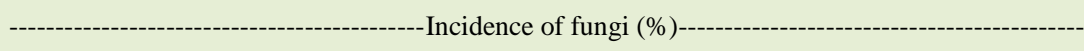 } \\
\hline & & Mic & Alt & Cur & Bip & Sar & Nig & Pen & Asp & Altsp & $\mathrm{Cer}$ & Fus \\
\hline \multicolumn{13}{|c|}{ 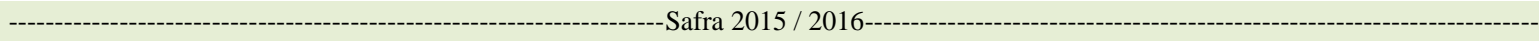 } \\
\hline SCS121 CL & 57 & 61.9 & 6.0 & 6.6 & 7.3 & 2.2 & 0.8 & 1.1 & 0.7 & 0.1 & 0.2 & 0.0 \\
\hline SCS116 Satoru & 33 & 72.3 & 2.9 & 1.7 & 1.6 & 2.2 & 1.6 & 1.3 & 1.2 & 0.4 & 0.3 & 0.0 \\
\hline SCSBRS Tio Taka & 17 & 55.6 & 7.7 & 5.7 & 7.3 & 2.7 & 3.0 & 0.5 & 0.7 & 0.9 & 0.4 & 0.4 \\
\hline Epagri 109 & 11 & 68.1 & 2.0 & 2.0 & 2.5 & 3.9 & 1.2 & 2.1 & 2.3 & 1.0 & 0.0 & 0.0 \\
\hline SCS117 CL & 11 & 60.5 & 9.0 & 3.9 & 5.1 & 2.5 & 1.4 & 0.4 & 0.3 & 0.5 & 0.4 & 0.0 \\
\hline SCS118 Marquês & 10 & 57.2 & 7.6 & 9.6 & 3.3 & 3.7 & 0.7 & 1.8 & 1.8 & 0.0 & 0.2 & 0.0 \\
\hline Average (\%) & & 62.6 & 5.8 & 4.9 & 4.5 & 2.8 & 1.4 & 1.2 & 1.2 & 0.5 & 0.3 & 0.1 \\
\hline \multicolumn{13}{|c|}{--1 } \\
\hline SCS121 CL & 55 & 530 & 7.4 & 3.0 & 2.7 & 4.2 & 0.9 & 0.6 & 1.0 & 0.7 & 1.2 & 0.1 \\
\hline SCS116 Satoru & 33 & 506 & 9.1 & 2.4 & 2.8 & 2.7 & 0.7 & 1.3 & 1.9 & 0.9 & 0.8 & 0.2 \\
\hline Epagri 109 & 31 & 455 & 11.3 & 3.0 & 3.2 & 3.9 & 0.6 & 0.8 & 1.3 & 1.5 & 0.6 & 0.3 \\
\hline SCSBRS Tio Taka & 26 & 627 & 6.5 & 2.5 & 3.4 & 3.4 & 0.7 & 0.5 & 0.7 & 1.0 & 0.5 & 0.3 \\
\hline SCS122 Miura & 12 & 431 & 11.7 & 5.6 & 5.1 & 7.1 & 0.0 & 1.2 & 2.1 & 2.3 & 1.1 & 0.0 \\
\hline SCS118 Marquês & 12 & 453 & 9.3 & 3.0 & 3.3 & 4.8 & 1.6 & 0.4 & 0.8 & 1.6 & 1.1 & 0.0 \\
\hline Average (\%) & & 500 & 9.2 & 3.2 & 3.4 & 4.3 & 0.7 & 0.8 & 1.3 & 1.3 & 0.9 & 0.2 \\
\hline \multicolumn{13}{|c|}{ 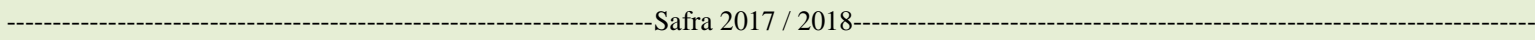 } \\
\hline SCS122 Miura & 73 & 535 & 7.2 & 1.7 & 1.6 & 1.1 & 0.6 & 0.1 & 0.0 & 0.3 & 1.2 & 0.0 \\
\hline SCS121 CL & 53 & 516 & 5.9 & 0.8 & 3.3 & 0.5 & 0.8 & 0.1 & 0.2 & 0.4 & 1.0 & 0.1 \\
\hline Epagri 109 & 16 & 446 & 14.3 & 1.7 & 1.9 & 0.3 & 0.7 & 0.1 & 0.0 & 0.8 & 1.2 & 0.0 \\
\hline SCS116 Satoru & 13 & 489 & 8.9 & 1.2 & 2.6 & 0.3 & 0.0 & 0.2 & 0.4 & 0.7 & 2.5 & 0.1 \\
\hline Primoriso CL & 10 & 577 & 7.2 & 1.0 & 0.9 & 0.9 & 0.5 & 0.2 & 0.0 & 0.4 & 2.8 & 0.0 \\
\hline SCSBRS Tio Taka & 6 & 562 & 4.5 & 0.7 & 2.5 & 2.2 & 0.3 & 0.3 & 0.0 & 0.3 & 1.2 & 0.0 \\
\hline Average (\%) & & 521 & 8.0 & 1.2 & 2.1 & 0.9 & 0.5 & 0.2 & 0.1 & 0.5 & 1.6 & 0.0 \\
\hline Overall Average (\%) & & 550 & 7.7 & 3.1 & 3.3 & 2.7 & 0.9 & 0.7 & 0.9 & 0.8 & 0.9 & 0.1 \\
\hline
\end{tabular}

Mic - Microdochium albescens; Alt - Alternaria padwiicki; Cur - Curvularia sp.; Bip - Bipolaris oryzae; Sar - Sarocladium oryzae; Nig - Nigrospora oryza; Pen - Penicillium spp.; Asp - Aspergillus flavus; Alt ${ }_{\mathrm{sp}}$ - Alternaria sp.; Cer - Cercospora janseana e Fus Fusarium sp. 


\section{Read:}

Table 1 - Average incidence of fungi associated with seeds of irrigated rice cultivars produced in the Alto Vale do Itajaí Region, Santa Catarina State. Lages, 2019.

\begin{tabular}{|c|c|c|c|c|c|c|c|c|c|c|c|c|}
\hline \multirow[t]{2}{*}{ Cultivar } & \multirow[t]{2}{*}{$\mathrm{N}^{\mathrm{o}}$ Lotes } & \multicolumn{11}{|c|}{ - Incidence of fungi $(\%)-$} \\
\hline & & Mic & Alt & Cur & Bip & Sar & $\mathrm{Nig}$ & Pen & Asp & Altsp & $\mathrm{Cer}$ & Fus \\
\hline & & & & Safra 2 & $15 / 201$ & & & & & & & ----- \\
\hline SCS121 CL & 57 & 61.9 & 6.0 & 6.6 & 7.3 & 2.2 & 0.8 & 1.1 & 0.7 & 0.1 & 0.2 & 0.0 \\
\hline SCS116 Satoru & 33 & 72.3 & 2.9 & 1.7 & 1.6 & 2.2 & 1.6 & 1.3 & 1.2 & 0.4 & 0.3 & 0.0 \\
\hline SCSBRS Tio Taka & 17 & 55.6 & 7.7 & 5.7 & 7.3 & 2.7 & 3.0 & 0.5 & 0.7 & 0.9 & 0.4 & 0.4 \\
\hline Epagri 109 & 11 & 68.1 & 2.0 & 2.0 & 2.5 & 3.9 & 1.2 & 2.1 & 2.3 & 1.0 & 0.0 & 0.0 \\
\hline SCS117 CL & 11 & 60.5 & 9.0 & 3.9 & 5.1 & 2.5 & 1.4 & 0.4 & 0.3 & 0.5 & 0.4 & 0.0 \\
\hline SCS118 Marquês & 10 & 57.2 & 7.6 & 9.6 & 3.3 & 3.7 & 0.7 & 1.8 & 1.8 & 0.0 & 0.2 & 0.0 \\
\hline Average (\%) & & 62.6 & 5.8 & 4.9 & 4.5 & 2.8 & 1.4 & 1.2 & 1.2 & 0.5 & 0.3 & 0.1 \\
\hline ----- & 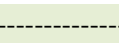 & - & - & Safra 20 & $6 / 201$ & - & ----- & - & ---- & - & ---- & ----- \\
\hline SCS121 CL & 55 & 53.0 & 7.4 & 3.0 & 2.7 & 4.2 & 0.9 & 0.6 & 1.0 & 0.7 & 1.2 & 0.1 \\
\hline SCS116 Satoru & 33 & 50.6 & 9.1 & 2.4 & 2.8 & 2.7 & 0.7 & 1.3 & 1.9 & 0.9 & 0.8 & 0.2 \\
\hline Epagri 109 & 31 & 45.5 & 11.3 & 3.0 & 3.2 & 3.9 & 0.6 & 0.8 & 1.3 & 1.5 & 0.6 & 0.3 \\
\hline SCSBRS Tio Taka & 26 & 62.7 & 6.5 & 2.5 & 3.4 & 3.4 & 0.7 & 0.5 & 0.7 & 1.0 & 0.5 & 0.3 \\
\hline SCS122 Miura & 12 & 43.1 & 11.7 & 5.6 & 5.1 & 7.1 & 0.0 & 1.2 & 2.1 & 2.3 & 1.1 & 0.0 \\
\hline SCS118 Marquês & 12 & 45.3 & 9.3 & 3.0 & 3.3 & 4.8 & 1.6 & 0.4 & 0.8 & 1.6 & 1.1 & 0.0 \\
\hline Average (\%) & & 50.0 & 9.2 & 3.2 & 3.4 & 4.3 & 0.7 & 0.8 & 1.3 & 1.3 & 0.9 & 0.2 \\
\hline ----------- & $\ldots$ & ---- & ------- & Safra 20 & $7 / 201$ & ------ & ----- & -- & ---- & --- & ---- & ----- \\
\hline SCS122 Miura & 73 & 53.5 & 7.2 & 1.7 & 1.6 & 1.1 & 0.6 & 0.1 & 0.0 & 0.3 & 1.2 & 0.0 \\
\hline SCS121 CL & 53 & 51.6 & 5.9 & 0.8 & 3.3 & 0.5 & 0.8 & 0.1 & 0.2 & 0.4 & 1.0 & 0.1 \\
\hline Epagri 109 & 16 & 44.6 & 14.3 & 1.7 & 1.9 & 0.3 & 0.7 & 0.1 & 0.0 & 0.8 & 1.2 & 0.0 \\
\hline SCS116 Satoru & 13 & 48.9 & 8.9 & 1.2 & 2.6 & 0.3 & 0.0 & 0.2 & 0.4 & 0.7 & 2.5 & 0.1 \\
\hline Primoriso CL & 10 & 57.7 & 7.2 & 1.0 & 0.9 & 0.9 & 0.5 & 0.2 & 0.0 & 0.4 & 2.8 & 0.0 \\
\hline SCSBRS Tio Taka & 6 & 56.2 & 4.5 & 0.7 & 2.5 & 2.2 & 0.3 & 0.3 & 0.0 & 0.3 & 1.2 & 0.0 \\
\hline Average (\%) & & 52.1 & 8.0 & 1.2 & 2.1 & 0.9 & 0.5 & 0.2 & 0.1 & 0.5 & 1.6 & 0.0 \\
\hline Overall Average (\%) & & 55.0 & 7.7 & 3.1 & 3.3 & 2.7 & 0.9 & 0.7 & 0.9 & 0.8 & 0.9 & 0.1 \\
\hline
\end{tabular}

Mic - Microdochium albescens; Alt - Alternaria padwiicki; Cur - Curvularia sp.; Bip - Bipolaris oryzae; Sar - Sarocladium oryzae; Nig - Nigrospora oryza; Pen - Penicillium spp.; Asp - Aspergillus flavus; Alt ${ }_{\mathrm{sp}}$ - Alternaria sp.; Cer - Cercospora janseana e Fus Fusarium sp.

\section{In the table 2, where we read:}

Table 2 - Average prevalence of fungi associated with seeds of irrigated rice cultivars produced in the Alto Vale do Itajaí Region, Santa Catarina State. Lages, 2019.

\begin{tabular}{|c|c|c|c|c|c|c|c|c|c|c|c|c|}
\hline \multirow[t]{2}{*}{ Cultivar } & \multirow[t]{2}{*}{$\mathrm{N}^{\mathrm{o}}$ Lotes } & \multicolumn{11}{|c|}{ - } \\
\hline & & Mic & Alt & Cur & Bip & Sar & Nig & Pen & Asp & Altsp & Cer & Fus \\
\hline & & & & $---S a f$ & 2015 & :016- & ------- & ------- & -------- & ------- & --------. & ------- \\
\hline SCS121 CL & 57 & 100 & 84.7 & 66.7 & 39.4 & 63.2 & 36.4 & 33.3 & 48.5 & 21.2 & 9.1 & 0.0 \\
\hline SCS116 Satoru & 33 & 100 & 100 & 70.6 & 76.5 & 57.6 & 64.7 & 29.4 & 23.5 & 17.7 & 17.7 & 0.0 \\
\hline SCSBRS Tio Taka & 17 & 100 & 100 & 90.9 & 45.5 & 64.7 & 54.6 & 36.4 & 9.1 & 18.2 & 18.2 & 0.4 \\
\hline Epagri 109 & 11 & 100 & 36.4 & 45.5 & 54.6 & 81.8 & 27.3 & 54.6 & 63.6 & 27.3 & 9.1 & 0.0 \\
\hline SCS117 CL & 11 & 100 & 89.5 & 89.5 & 77.2 & 72.7 & 26.3 & 45.6 & 22.8 & 8.8 & 14.0 & 0.0 \\
\hline SCS118 Marquês & 10 & 100 & 90.0 & 100 & 50.0 & 70.0 & 40.0 & 50.0 & 40.0 & 0.0 & 100 & 0.0 \\
\hline Average (\%) & & 100 & 83.4 & 77.2 & 57.2 & 68.3 & 41.6 & 41.6 & 34.6 & 15.5 & 13.0 & 0.1 \\
\hline ---------------. & 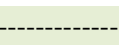 & 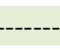 & ------- & -Safra & $016 / 2$ & 7----- & ------- & ----- & ------ & ----- & ------ & ----- \\
\hline SCS121 CL & 55 & 100 & 89.1 & 80.0 & 63.6 & 63.6 & 23.6 & 27.3 & 36.4 & 32.7 & 40.0 & 3.6 \\
\hline SCS116 Satoru & 33 & 100 & 90.9 & 69.7 & 60.6 & 45.5 & 21.2 & 48.5 & 42.4 & 36.4 & 21.2 & 6.1 \\
\hline Epagri 109 & 31 & 100 & 92.3 & 69.2 & 53.8 & 38.5 & 19.2 & 21.9 & 19.2 & 46.2 & 34.6 & 7.7 \\
\hline SCSBRS Tio Taka & 26 & 100 & 96.8 & 77.4 & 67.7 & 58.1 & 16.1 & 32.3 & 45.2 & 54.8 & 32.3 & 9.7 \\
\hline SCS122 Miura & 12 & 100 & 100 & 91.7 & 100 & 91.7 & 0.0 & 58.3 & 50.0 & 75.0 & 33.3 & 16.7 \\
\hline
\end{tabular}




\begin{tabular}{|c|c|c|c|c|c|c|c|c|c|c|c|c|}
\hline SCS118 Marquês & 12 & 100 & 100 & 91.7 & 75.0 & 50.0 & 50.0 & 16.2 & 25.0 & 50.0 & 25.0 & 16.7 \\
\hline Average (\%) & & 100 & 94.8 & 79.9 & 70.1 & 57.9 & 21.7 & 34.1 & 36.4 & 49.2 & 31.1 & 10.1 \\
\hline \multicolumn{13}{|c|}{ 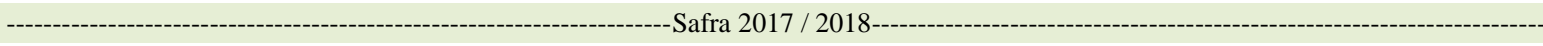 } \\
\hline SCS122 Miura & 73 & 100 & 868 & 26.4 & 66.0 & 18.9 & 22.6 & 5.7 & 7.6 & 18.9 & 32.1 & 5.7 \\
\hline SCS121 CL & 53 & 100 & 100 & 53.1 & 69.2 & 15.4 & 23.1 & 15.4 & 15.4 & 30.8 & 53.8 & 0.0 \\
\hline Epagri 109 & 16 & 100 & 100 & 16.6 & 50.0 & 0.0 & 16.7 & 16.7 & 0.0 & 16.7 & 50.0 & 0.0 \\
\hline SCS116 Satoru & 13 & 100 & 937 & 50.0 & 56.3 & 12.5 & 18.8 & 6.3 & 0.0 & 31.3 & 50.0 & 0.0 \\
\hline Primoriso CL & 10 & 100 & 918 & 57.5 & 45.2 & 34.3 & 12.3 & 6.9 & 1.4 & 10.9 & 49.3 & 2.7 \\
\hline SCSBRS Tio Taka & 6 & 100 & 90.0 & 50.0 & 30.0 & 50.0 & 0.0 & 10.0 & 0.0 & 20.0 & 90.0 & 0.0 \\
\hline Average (\%) & & 100 & 93.7 & 42.3 & 52.8 & 21.9 & 15.6 & 10.2 & 4.1 & 21.4 & 54.2 & 1.4 \\
\hline Overall Average (\%) & & 100 & 90.6 & 66.5 & 59.9 & 49.4 & 26.3 & 28.6 & 25.0 & 28.7 & 32.8 & 11.6 \\
\hline
\end{tabular}

Mic - Microdochium albescens; Alt - Alternaria padwiicki; Cur - Curvularia sp.; Bip - Bipolaris oryzae; Sar - Sarocladium oryzae; Nig - Nigrospora oryza; Pen - Penicillium spp.; Asp - Aspergillus flavus; Alt ${ }_{\mathrm{sp}}$ - Alternaria sp.; Cer - Cercospora janseana e Fus Fusarium sp.

\section{Read:}

Table 2 - Average prevalence of fungi associated with seeds of irrigated rice cultivars produced in the Alto Vale do Itajaí Region, Santa Catarina State. Lages, 2019.

\begin{tabular}{|c|c|c|c|c|c|c|c|c|c|c|c|c|}
\hline \multirow[t]{2}{*}{ Cultivar } & \multirow[t]{2}{*}{$\mathrm{N}^{\mathrm{o}}$ Lotes } & \multicolumn{11}{|c|}{ 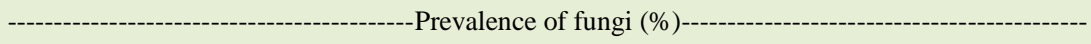 } \\
\hline & & Mic & Alt & Cur & Bip & Sar & Nig & Pen & Asp & Altsp & Cer & Fus \\
\hline \multicolumn{13}{|c|}{ 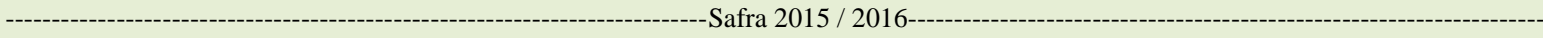 } \\
\hline SCS121 CL & 57 & 100 & 84.7 & 66.7 & 39.4 & 63.2 & 36.4 & 33.3 & 48.5 & 21.2 & 9.1 & 0.0 \\
\hline SCS116 Satoru & 33 & 100 & 100 & 70.6 & 76.5 & 57.6 & 64.7 & 29.4 & 23.5 & 17.7 & 17.7 & 0.0 \\
\hline SCSBRS Tio Taka & 17 & 100 & 100 & 90.9 & 45.5 & 64.7 & 54.6 & 36.4 & 9.1 & 18.2 & 18.2 & 0.4 \\
\hline Epagri 109 & 11 & 100 & 36.4 & 45.5 & 54.6 & 81.8 & 27.3 & 54.6 & 63.6 & 27.3 & 9.1 & 0.0 \\
\hline SCS117 CL & 11 & 100 & 89.5 & 89.5 & 77.2 & 72.7 & 26.3 & 45.6 & 22.8 & 8.8 & 14.0 & 0.0 \\
\hline SCS118 Marquês & 10 & 100 & 90.0 & 100 & 50.0 & 70.0 & 40.0 & 50.0 & 40.0 & 0.0 & 100 & 0.0 \\
\hline Average (\%) & & 100 & 83.4 & 77.2 & 57.2 & 68.3 & 41.6 & 41.6 & 34.6 & 15.5 & 13.0 & 0.1 \\
\hline \multicolumn{13}{|c|}{ 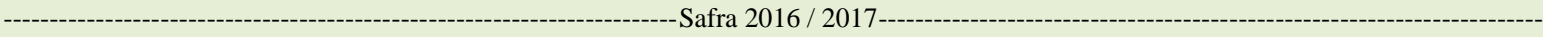 } \\
\hline SCS121 CL & 55 & 100 & 89.1 & 80.0 & 63.6 & 63.6 & 23.6 & 27.3 & 36.4 & 32.7 & 40.0 & 3.6 \\
\hline SCS116 Satoru & 33 & 100 & 90.9 & 69.7 & 60.6 & 45.5 & 21.2 & 48.5 & 42.4 & 36.4 & 21.2 & 6.1 \\
\hline Epagri 109 & 31 & 100 & 92.3 & 69.2 & 53.8 & 38.5 & 19.2 & 21.9 & 19.2 & 46.2 & 34.6 & 7.7 \\
\hline SCSBRS Tio Taka & 26 & 100 & 96.8 & 77.4 & 67.7 & 58.1 & 16.1 & 32.3 & 45.2 & 54.8 & 32.3 & 9.7 \\
\hline SCS122 Miura & 12 & 100 & 100 & 91.7 & 100 & 91.7 & 0.0 & 58.3 & 50.0 & 75.0 & 33.3 & 16.7 \\
\hline SCS118 Marquês & 12 & 100 & 100 & 91.7 & 75.0 & 50.0 & 50.0 & 16.2 & 25.0 & 50.0 & 25.0 & 16.7 \\
\hline Average (\%) & & 100 & 94.8 & 79.9 & 70.1 & 57.9 & 21.7 & 34.1 & 36.4 & 49.2 & 31.1 & 10.1 \\
\hline \multicolumn{13}{|c|}{ - } \\
\hline SCS122 Miura & 73 & 100 & 86.8 & 26.4 & 66.0 & 18.9 & 22.6 & 5.7 & 7.6 & 18.9 & 32.1 & 5.7 \\
\hline SCS121 CL & 53 & 100 & 100 & 53.1 & 69.2 & 15.4 & 23.1 & 15.4 & 15.4 & 30.8 & 53.8 & 0.0 \\
\hline Epagri 109 & 16 & 100 & 100 & 16.6 & 50.0 & 0.0 & 16.7 & 16.7 & 0.0 & 16.7 & 50.0 & 0.0 \\
\hline SCS116 Satoru & 13 & 100 & 93.7 & 50.0 & 56.3 & 12.5 & 18.8 & 6.3 & 0.0 & 31.3 & 50.0 & 0.0 \\
\hline Primoriso CL & 10 & 100 & 91.8 & 57.5 & 45.2 & 34.3 & 12.3 & 6.9 & 1.4 & 10.9 & 49.3 & 2.7 \\
\hline SCSBRS Tio Taka & 6 & 100 & 90.0 & 50.0 & 30.0 & 50.0 & 0.0 & 10.0 & 0.0 & 20.0 & 90.0 & 0.0 \\
\hline Average (\%) & & 100 & 93.7 & 42.3 & 52.8 & 21.9 & 15.6 & 10.2 & 4.1 & 21.4 & 54.2 & 1.4 \\
\hline Overall Average (\%) & & 100 & 90.6 & 66.5 & 59.9 & 49.4 & 26.3 & 28.6 & 25.0 & 28.7 & 32.8 & 11.6 \\
\hline
\end{tabular}

Mic - Microdochium albescens; Alt - Alternaria padwiicki; Cur - Curvularia sp.; Bip - Bipolaris oryzae; Sar - Sarocladium oryzae;

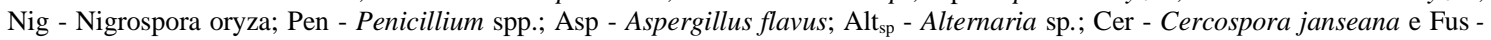
Fusarium sp. 